\title{
Analytical prediction of syngas induction times
}

\author{
P. Boivin ${ }^{1 a}$, A. L. Sánchez ${ }^{\mathrm{b}}$, F. A. Williams ${ }^{\mathrm{b}}$ \\ ${ }^{a}$ Aix Marseille Univ, CNRS, Centrale Marseille, M2P2, Marseille, France \\ ${ }^{b}$ Dept. of Mechanical and Aerospace Engineering, University of California San Diego, La \\ Jolla CA 92093-0411, USA
}

\begin{abstract}
Computations indicate that, under all possible conditions of practical interest, including temperatures both above and below the crossover temperature at which the rates of the $\mathrm{H}_{2}+\mathrm{O}_{2}$ branching and termination steps are equal, twelve irreversible elementary steps suffice to provide accurate values of induction times in autoignition processes of fuels consisting of mixtures of $\mathrm{H}_{2}$, $\mathrm{CO}$, and inerts. At high temperatures, this time is controlled by the time required for the radical pool to reach a steady state, with heat release being negligible during that time. This time is approximated well by the time that it would take for $\mathrm{HO}_{2}$ to reach a steady state if its consumption rate were dominated by formation of $\mathrm{H}_{2} \mathrm{O}_{2}$ at all temperatures, as it is at low temperatures. Below the crossover temperature, the time to reach an $\mathrm{HO}_{2}$ steady state becomes shorter than the induction time, and the heat release becomes non-negligible once $\mathrm{HO}_{2}$ has reached a steady state, resulting in the induction time progressively approaching that of a thermal explosion, which includes an effectively autocatalytic production of $\mathrm{H}_{2} \mathrm{O}_{2}$. On the basis of these observations, analytical approximations are introduced here that
\end{abstract}

\footnotetext{
${ }^{1}$ Corresponding author: pierre.boivin@univ-amu.fr
} 
enable induction times to be calculated accurately under all conditions. Keywords: combustion, syngas, hydrogen, autoignition

\section{Introduction}

There is increasing interest in the use of fuels produced synthetically, for example by gasification of coal or of biomass, in combustion systems such as gas turbines [1] and internal combustion engines [2]. These syngas fuels usually are predominantly mixtures of hydrogen, carbon monoxide, and inert constituents, although they often contain appreciable amounts of methane and other hydrocarbons [3]. Even when these additional species are present, however, the combustion characteristics of the syngas often can be described reasonably well by the combustion chemistry of mixtures of $\mathrm{H}_{2}$ and $\mathrm{CO}$. A number of detailed chemical-kinetic mechanisms for the combustion of such mixtures are available in the literature [4-8]. These mechanisms, which may still have inaccuracies at high pressures and low temperatures but nevertheless produce reasonable agreements with measurements in most respects, typically involve on the order of fifty or more elementary steps, which is large enough to cause them to be expensive or impossible to employ in certain applications involving computational fluid dynamics (CFD). It is therefore worthwhile to investigate the development of simpler approaches for solving specific related problems.

One problem, of special importance in gas-turbine applications, for example, is the autoignition problem. There is, in particular, considerable interest in ignition-delay times, or so-called induction times, in connection with flashback concerns for gas turbines operating on syngas. The combus- 
tion in properly operating gas turbines must be confined to the combustion chamber, but, especially when the fuel is syngas of high hydrogen content, autoignition may occur upstream, causing the combustion to flash back into the feed stream, sometimes with catastrophic results [9]. This phenomenon arises when the induction time is shorter than the residence time of the gas mixtures in the feed streams. Under idealized condition, the induction time can be defined as the time required for a uniform fuel-oxidizer gas mixture, placed at time zero in an isobaric or isochoric, adiabatic chamber, to begin to release heat rapidly. Such an idealized definition remains ambiguous until a specific definition is given for the phrase "begin to release heat rapidly". There are many different possible definitions for this phrase, all of which correspond to different values of the induction time, but under a wide range of conditions of practical interest, once a small amount of heat release occurs, the rate of heat release accelerates very rapidly, so that the different definitions produce induction-time values that differ very little. The present contribution employs the time of maximum rate of heat release as the ignition-time definition, but the result was found to be the same as the time required for the temperature to increase by $200 \mathrm{~K}$ or achieving a rate of temperature rise greater than $10,000 \mathrm{~K} / \mathrm{s}$.

In real-world scenarios, such as those that occur during the gas-turbine flashback mentioned above, mixture compositions and temperatures vary in space and time, so that a single idealized induction time is not directly relevant. The complete, detailed, chemical-kinetic mechanisms, however, are not needed if interest resides solely in the autoignition problem in these cases. The elementary chemistry that successfully describes the idealized in- 
duction times over the complete range of conditions experienced by the gas in the practical situation also can correctly predict the autoignition in the application, through appropriate CFD, when the spatial and temporal variations are taken into account. It therefore is worthwhile to identify simplified chemistry with fewer elementary reactions that is successful in wide-range induction-time predictions. This has been done in the present work. The following section presents a short chemical-kinetic mechanism, consisting of only twelve irreversible elementary steps, which predicts induction times that agree over all conditions considered with those obtained with a 30-step detailed mechanism. The reaction rates of the short mechanism involve the concentrations of $\mathrm{H}_{2}, \mathrm{CO}, \mathrm{O}_{2}, \mathrm{H}, \mathrm{O}, \mathrm{OH}, \mathrm{HO}_{2}$, and $\mathrm{H}_{2} \mathrm{O}_{2}$, but are practically independent of the concentrations of $\mathrm{CO}_{2}$ and $\mathrm{H}_{2} \mathrm{O}$, which appear only indirectly through the chaperon efficiencies of the three-body recombination reactions. Since consumption of reactants $\mathrm{H}_{2}, \mathrm{CO}$, and $\mathrm{O}_{2}$ and production of products $\mathrm{CO}_{2}$ and $\mathrm{H}_{2} \mathrm{O}$ are small during ignition, the number of reactingspecies equations that need to be considered is reduced from 10 to 5, and, together with the decrease in the number of elementary steps, this has been found in our representative computational investigations to result in a reduction of the associated computational time by about $30 \%$, thereby facilitating many types of numerical investigations. It should be cautioned that this chemistry will not correctly predict the subsequent combustion, but it will be reliable for autoignition events, affording utility in associated design and diagnostic tasks.

It is also of interest to have explicit analytical formulas for the idealized induction times. Such formulas are helpful, for example, in making 
preliminary estimates of autoignition processes, prior to CFD investigations. Such formulas have often been derived before under restricted conditions $[10$ 12]. In general, however, those restricted conditions cannot be guaranteed to be encountered in applications, and therefore formulas with wider ranges of validity are desired. By use of our simplified chemistry, such wide-range induction-time formulas are derived here for isobaric, adiabatic conditions. This entails identifying both the controlling autoignition processes over the full range of conditions and corresponding useful specific definitions of induction times. Those developments are presented in subsequent sections of the paper.

The results for the homogeneous ignition problem addressed here can be useful in understanding more complex scenarios, including the nonuniform time-varying environments found in reciprocating engines, where conditions evolve from the low-temperature to the high-temperature regimes identified below, necessarily complicating the required analysis. Although the results presented specifically pertain to mixtures of $\mathrm{CO}-\mathrm{H}_{2}$ with air, all of the conclusions, as well as the analytic formulae derived, apply also to ignition employing pure $\mathrm{O}_{2}$ or general $\mathrm{O}_{2}$-inert oxidizer mixtures with various degrees of dilution.

\section{Simplified chemistry for syngas ignition}

Table 1 lists the elementary steps with their associated rate parameters for a 12-step mechanism that describes syngas chemistry during ignition as accurately as any detailed mechanism. The values of the rate parameters in the table are taken from the San Diego mechanism [13], which includes recently updated rate information for the first eight steps [14]. Figure 11 compares 
predictions of induction times by this mechanism with predictions obtained with the corresponding full detailed mechanism for a range of conditions, including those for which the predictions from Table 1 are the worst. It is seen
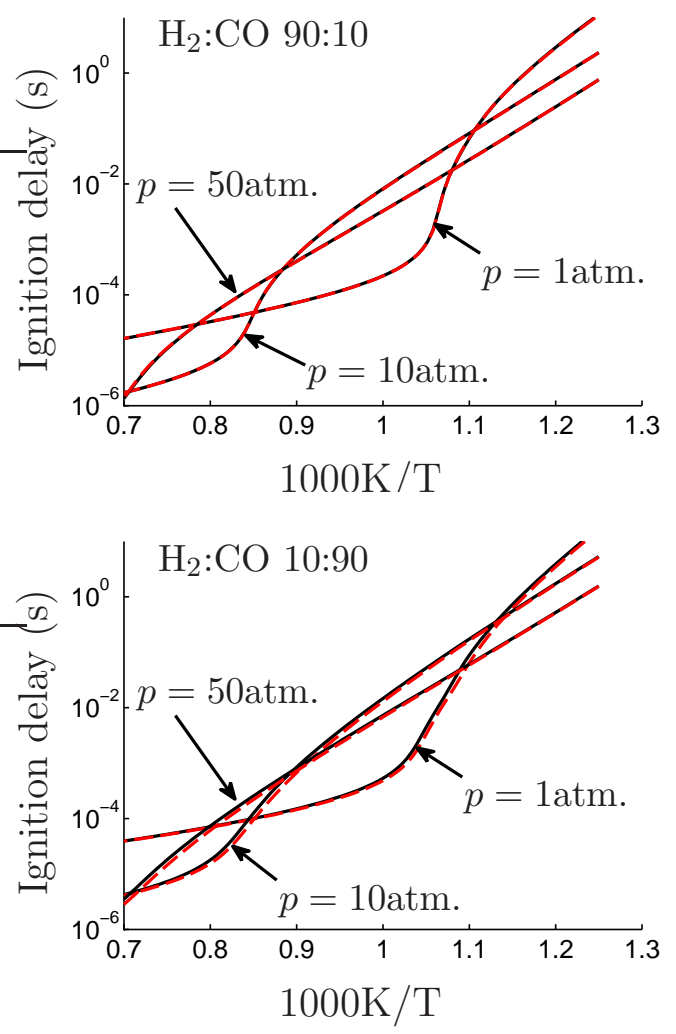

Figure 1: Comparisons of ignition delays computed with detailed chemistry (solid curves) and 12-step skeletal chemistry (dashed curves) for two stoichiometric syngas-air mixtures with different $\mathrm{CO}: \mathrm{H}_{2}$ relative composition.

from this figure that the differences are almost completely indistinguishable in all cases. This mechanism has been selected specifically to address ignition delay times, and other steps, as well as reverse reactions, become important in addressing other questions, especially at high temperatures. For the two mixtures in Fig. 1, at the same pressure and initial temperature, the ignition 
delays differ by a factor of order unity, an aspect of the problem to be further addressed in Fig. 9 .

\begin{tabular}{|c|c|c|c|c|c|}
\hline & Reaction & & $A^{a}$ & $n^{a}$ & $E^{a}$ \\
\hline 1 & $\mathrm{H}+\mathrm{O}_{2} \rightarrow \mathrm{OH}+\mathrm{O}$ & & $3.5210^{16}$ & -0.7 & 71.42 \\
\hline 2 & $\mathrm{H}_{2}+\mathrm{O} \rightarrow \mathrm{OH}+\mathrm{H}$ & & $5.0610^{4}$ & 2.67 & 26.32 \\
\hline 3 & $\mathrm{H}_{2}+\mathrm{OH} \rightarrow \mathrm{H}_{2} \mathrm{O}+\mathrm{H}$ & & $1.1710^{9}$ & 1.3 & 15.17 \\
\hline \multirow[t]{2}{*}{4} & $\mathrm{H}+\mathrm{O}_{2}+\mathrm{M} \rightarrow \mathrm{HO}_{2}+\mathrm{M}^{b}$ & $k_{0}$ & $5.7510^{19}$ & -1.4 & 0.0 \\
\hline & & $k_{\infty}$ & $4.6510^{12}$ & 0.44 & 0.0 \\
\hline 5 & $\mathrm{H}_{2}+\mathrm{O}_{2} \rightarrow \mathrm{HO}_{2}+\mathrm{H}$ & & $2.9310^{12}$ & 0.356 & 232.21 \\
\hline $6^{c}$ & $2 \mathrm{HO}_{2} \rightarrow \mathrm{H}_{2} \mathrm{O}_{2}+\mathrm{O}_{2}$ & & $\begin{array}{l}1.0310^{14} \\
1.9410^{11}\end{array}$ & $\begin{array}{l}0.0 \\
0.0\end{array}$ & $\begin{array}{l}46.22 \\
-5.89\end{array}$ \\
\hline 7 & $\mathrm{HO}_{2}+\mathrm{H}_{2} \rightarrow \mathrm{H}_{2} \mathrm{O}_{2}+\mathrm{H}$ & & $7.8010^{10}$ & 0.61 & 100.14 \\
\hline \multirow[t]{2}{*}{8} & $\mathrm{H}_{2} \mathrm{O}_{2}+\mathrm{M} \rightarrow 2 \mathrm{OH}+\mathrm{M}^{d}$ & $k_{0}$ & $7.6010^{30}$ & -4.20 & 213.71 \\
\hline & & $k_{\infty}$ & $2.6310^{19}$ & -1.27 & 214.74 \\
\hline 9 & $\mathrm{CO}+\mathrm{OH} \rightarrow \mathrm{CO}_{2}+\mathrm{H}$ & & $4.4010^{6}$ & 1.5 & -3.1 \\
\hline 10 & $\mathrm{CO}+\mathrm{HO}_{2} \rightarrow \mathrm{CO}_{2}+\mathrm{OH}$ & & $2.0010^{13}$ & 0.0 & 96.0 \\
\hline 11 & $\mathrm{CO}+\mathrm{O}_{2} \rightarrow \mathrm{CO}_{2}+\mathrm{O}$ & & $1.0010^{12}$ & 0.00 & 199.57 \\
\hline \multirow[t]{2}{*}{12} & $\mathrm{CO}+\mathrm{O}+\mathrm{M} \rightarrow \mathrm{CO}_{2}+\mathrm{M}^{e}$ & $k_{0}$ & $1.5510^{24}$ & -2.79 & 17.55 \\
\hline & & $k_{\infty}$ & $1.810^{11}$ & 0.0 & 23.8 \\
\hline
\end{tabular}

Table 1: Rate coefficients responsible for syngas ignition in Arrhenius form $k=$ $A T^{n} \exp \left(-E / R^{o} T\right)$ for the skeletal mechanism, with numerical values of the San Diego mechanism [13].

${ }^{a}$ Units are mol, $\mathrm{s}, \mathrm{cm}^{3}, \mathrm{~kJ}$, and $\mathrm{K}$.

${ }^{b}$ Chaperon efficiencies are 2.5 for $\mathrm{H}_{2}, 16.0$ for $\mathrm{H}_{2} \mathrm{O}, 0.7$ for Ar and $\mathrm{He}$ and 1.0 for all other species; Troe falloff with $F_{c}=0.5$

${ }^{c}$ Bi-Arrhenius (the sum of the two constants).

${ }^{d}$ Chaperon efficiencies are 2.0 for $\mathrm{H}_{2}, 6.0$ for $\mathrm{H}_{2} \mathrm{O}, 0.4$ for $\mathrm{Ar}$ and $\mathrm{He}$ and 1.0 for all other species; $F_{c}=0.265 \exp (-T / 94 \mathrm{~K})+0.735 \exp (-T / 1756 \mathrm{~K})+\exp (-5182 \mathrm{~K} / T)$

${ }^{e}$ Chaperon efficiencies are 2.5 for $\mathrm{H}_{2}, 12.0$ for $\mathrm{H}_{2} \mathrm{O}, 2.0$ for $\mathrm{CO}, 4$ for $\mathrm{CO}_{2}, 0.4$ for Ar and He and 1.0 for all other species; $F_{c}=\exp \left(-T / 10^{7} \mathrm{~K}\right)+\exp \left(-10^{7} \mathrm{~K} / T\right)$

The first 8 steps in Table 1 are the steps found in our previous work [12, 15, 16] to have to be retained for hydrogen autoignition. An initiation step to produce radicals from the reactants is always necessary, and this appears as step 5 in the table for hydrogen. In syngas, initiation also can occur from carbon monoxide, and that step appears as step 11 in the table. The 
first three entries in the table are hydrogen-oxygen chain-carrying steps (the first two chain-branching), the so-called hydrogen-oxygen shuffle reactions; there are four such elementary reactions, but, in autoignition, the fourth shuffle reaction $\left(\mathrm{OH}+\mathrm{OH} \rightleftharpoons \mathrm{H}_{2} \mathrm{O}+\mathrm{O}\right)$ does not have to be retained when the first three are included. The fourth step in the table is the important step for removal of the most active radicals that carry the high-temperature hydrogen-oxygen branched-chain reaction mechanism, the mechanism that is dominant when the temperature exceeds the crossover temperature at which the rate of the fourth step is twice that of the first [17]. For syngas, in this high-temperature range, the straight-chain steps 9 and 10 need to be retained for both chemical-kinetic and energetic reasons, and step 12 becomes an important step for removing the most active radicals, like step 4 . The competitions between step 3 and 9 (consumption of $\mathrm{OH}$ radical by $\mathrm{H}_{2}$ and $\mathrm{CO}$ ), and between steps 2 and 12 (consumption of $\mathrm{O}$ radical by $\mathrm{H}_{2}$ and $\mathrm{CO}$ ) are fundamental in describing the branched-chain reaction mechanism. At temperatures below crossover, the branched-chain mechanism of steps 1-3 is quenched by the dominant radical-recombination path of reaction 4 , and the hydroperoxyl steps 6 and 7 then become important for producing autoignition through the generation of active radicals in step 8 .

\section{The Chain-Branching Stage}

\subsection{Isothermal branched-chain radical growth}

In the absence of radicals in the initial mixture, the homogeneous ignition history always displays a stage of rapid exponential radical growth following a short initial period of slow radical generation controlled the initiation steps 5 and 11 . Since both reactant consumption and heat release 
have a secondary effect over most of this chain-branching period, the radical conservation equations can be simplified by evaluating the chemical rates at the initial temperature and initial reactant concentrations, that being the approach taken in analytical studies of high-temperature hydrogen-oxygen ignition [18] (see also the early references cited in [19]). Furthermore, since the radical concentrations are small compared with those of the reactants, the effect of reaction 6 , involving the collision of two radicals, can also be neglected, thereby reducing the problem to that of integrating the linear system of equations

$$
\frac{\mathrm{d} \bar{C}}{\mathrm{~d} t}=\mathbf{A} \bar{C}+\bar{\epsilon}
$$

where

$$
\bar{C}=\left[\begin{array}{lllll}
C_{\mathrm{H}} & C_{\mathrm{O}} & C_{\mathrm{OH}} & C_{\mathrm{HO}_{2}} & C_{\mathrm{H}_{2} \mathrm{O}_{2}}
\end{array}\right]^{\top}
$$

is the radical-concentration vector,

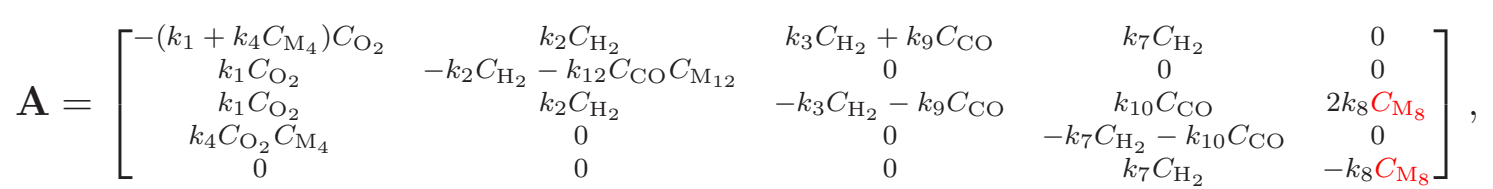

is the Jacobian matrix corresponding to the chain-branching chemistry, and

$$
\bar{\epsilon}=\left[\begin{array}{lllll}
\omega_{5} & \omega_{11} & 0 & \omega_{5} & 0
\end{array}\right]^{\top}
$$

is the vector containing the initiation rates $\omega_{5}=k_{5} C_{\mathrm{O}_{2}} C_{\mathrm{H}_{2}}$ and $\omega_{11}=$ $k_{11} C_{\mathrm{O}_{2}} C_{\mathrm{CO}}$. Here, $C_{i}$ represents the concentration of species $i$, while $C_{\mathrm{M}_{j}}$ is the effective third-body concentration of step $j=(4,8,12)$. In the following, we introduce inverse characteristic times for each reaction $l_{1}=k_{1} C_{\mathrm{O}_{2}}$, 
$l_{2}=k_{2} C_{\mathrm{H}_{2}}$ and so on, simplifying the notation appreciably, so that

$$
\mathbf{A}=\left[\begin{array}{ccccc}
-l_{1}-l_{4} & l_{2} & l_{3}+l_{9} & l_{7} & 0 \\
l_{1} & -l_{2}-l_{12} & 0 & 0 & 0 \\
l_{1} & l_{2} & -l_{3}-l_{9} & l_{10} & 2 l_{8} \\
l_{4} & 0 & 0 & -l_{7}-l_{10} & 0 \\
0 & 0 & 0 & l_{7} & -l_{8}
\end{array}\right]
$$

The solution to (1) with initial conditions $\bar{C}(0)=0$ can be cast in the form

$$
\bar{C}=\sum_{i=1,5} a_{i} \bar{V}_{i} e^{\lambda_{i} t}+\bar{C}_{0},
$$

involving the eigenvalues $\lambda_{i}$ and associated eigenvectors $\bar{V}_{i}$ of the Jacobian matrix $\mathbf{A}$ along with the particular solution $\bar{C}_{0}$, obtained by solving $\mathbf{A} \bar{C}_{0}+\bar{\epsilon}=$ 0 . The coefficients $a_{i}$ are determined by imposing the initial (null) condition $\sum_{i=1,5} a_{i} \bar{V}_{i}+\bar{C}_{0}=0$.

\subsection{The duration of the branching stage}

The set of eigenvalues $\lambda_{i}$, obtained as solutions to the characteristic equation associated with $\mathbf{A}$, includes one or more positive real values. Because of the exponential growth of the solution, the largest eigenvalue, denoted by $\lambda$ with associated coefficient $a$ and eigenvector $\bar{V}=\left(V_{\mathrm{H}}, V_{\mathrm{O}}, V_{\mathrm{OH}}, V_{\mathrm{HO}_{2}}, V_{\mathrm{H}_{2} \mathrm{O}_{2}}\right)$, soon becomes dominant, so that (6) simplifies for $\lambda t \gg 1$ to

$$
\bar{C}=a \bar{V} e^{\lambda t}
$$

This equation provides a sufficiently accurate description of the intermediatespecies evolution during the first stages of ignition, irrespective of whether the temperature is above or below crossover. This is shown in Fig. 2, which compares results of numerical integrations with the predictions obtained from (7) for the H-atom mole fraction $X_{\mathrm{H}}=\left(a V_{\mathrm{H}} e^{\lambda t}\right) /\left[p /\left(R^{o} T\right)\right]$ and $\mathrm{HO}_{2}$ mole fraction $X_{\mathrm{HO}_{2}}=\left(a V_{\mathrm{HO}_{2}} e^{\lambda t}\right) /\left[p /\left(R^{o} T\right)\right]$. 
Figure 2: $\mathrm{H}$ and $\mathrm{HO}_{2}$ mole fractions and temperature as functions of time, during isobaric homogeneous ignition processes from numerical integrations with the 12-step skeletal chemistry for $\phi=1, p=1 \mathrm{~atm}$, and $\mathrm{H}_{2}-\mathrm{CO}: 50-50$, with $T=1200 \mathrm{~K}$ (top) and $T=900 \mathrm{~K}$ (bottom); the $\mathrm{H}$ and $\mathrm{HO}_{2}$ mole fractions evaluated with use of Eq. (77) are shown as dashed lines.

This linearized approach remains valid until the rate of reaction 6 - the only one not included in this description - becomes important. From Eq. (7), it becomes straightforward to write the time at which this occurs as

$$
t_{B}=\lambda^{-1} \ln \left(\frac{\lambda}{2 a k_{6} V_{\mathrm{HO}_{2}}}\right),
$$

which defines the instant at which $\mathrm{HO}_{2}$ reaches a steady state, calculated by equating the $\mathrm{HO}_{2}$ production rate $\lambda a V_{\mathrm{HO}_{2}} e^{\lambda t}$ to its consumption rate by step 6, expressed in the form $2 k_{6} C_{\mathrm{HO}_{2}}^{2}=2 k_{6}\left(a V_{\mathrm{HO}_{2}} e^{\lambda t}\right)^{2}$, with $V_{\mathrm{HO}_{2}}$ denoting the fourth component of the eigenvector $\bar{V}$ associated with the largest real 
eigenvalue $\lambda$.

The time $t_{B}$ computed from (8) is selected in Fig. 2 as the end point for the lines evaluated from (7). As can be seen, at high temperature the H-atom mole fraction grows to significant values $\sim 0.1$ at the end of the branching stage, with the temperature beginning to increase appreciably for $t>t_{B}$ as a result of the subsequent radical recombination. Under those hightemperature conditions, therefore, the prediction for $t_{B}$ becomes a prediction for the induction time $t_{i}$. The behavior encountered at low temperatures is markedly different, as seen in Fig. 2 from the computations with $T=900$ $\mathrm{K}$. The radical concentration is negligibly small at the end of the branching period, which is followed by a stage of comparable duration ending with a rapid temperature increase. For low temperatures, therefore, the prediction of the induction time $t_{i}$ requires consideration of two different stages, the second of which, for $t_{B}<t<t_{i}$, being a thermal explosion occurring with all radicals in steady state. The thermal-explosion stage will be seen below to become dominant as the temperature decreases, so that for temperatures sufficiently below crossover the branching stage can be neglected in the first approximation in providing predictions for $t_{i}$, as done earlier for hydrogen-air ignition [12].

The relation between $t_{B}$ and the induction time is investigated in Fig. 3, which compares the value of $t_{i}$ determined from detailed-chemistry computations with the prediction for $t_{B}$ obtained from evaluations of (8). As can be seen, while both times are identical for temperatures exceeding about 950K, the crossover corresponding to atmospheric conditions, growing departures are found for lower temperatures, in agreement with the ignition histories 
Figure 3: Ignition delay $t_{i}$ (thick solid line) determined numerically with the 30-step San Diego mechanism for a stoichiometric syngas-air mixture with $\mathrm{H}_{2}$ :CO: 90:10 at atmospheric pressure. The thin solid line represents the branching time $t_{B}$ evaluated from Eq. (8), with the dashed lines representing the low-temperature and high-temperature predictions obtained from Eqs. (19) and (27) and the circles representing predictions obtained from Eq. (34).

shown in Fig. 2, The figure also includes different analytical predictions of $t_{B}$, to be developed below.

\subsection{The crossover temperature}

Although the coefficients $a$ and $\lambda$ in Eq. (8) cannot be obtained analytically for the $5 \times 5$ matrix system, analytical results can be derived by studying regimes above and below the crossover temperature. To facilitate these analytical studies, the parameter

$$
\delta_{\mathrm{O}}=\frac{k_{12} C_{\mathrm{M}_{12}} C_{\mathrm{CO}}}{k_{2} C_{\mathrm{H}_{2}}+k_{12} C_{\mathrm{M}_{12}} C_{\mathrm{CO}}}
$$

is introduced, representing the fraction of radicals $\mathrm{O}$ consumed by carbon monoxide (rather than by hydrogen). This parameter is 0 in pure hydrogen, and 1 in pure carbon monoxide. It is helpful to introduce an additional 
parameter defined as

$$
\alpha=\frac{2 l_{1} l_{2}}{l_{4}\left(l_{2}+l_{12}\right)}=\frac{2 k_{1}}{k_{4} C_{\mathrm{M}_{4}}}\left(1-\delta_{\mathrm{O}}\right),
$$

with the value $\alpha=1$ taken to define crossover, as will become clear from the following results. For pure hydrogen, $\delta_{\mathrm{O}}=0$, and $\alpha$ reduces to $2 k_{1} /\left(k_{4} C_{\mathrm{M}_{4}}\right)$, in agreement with the classical result [17] that, at crossover, the rate of reaction $\mathrm{O}_{2}+\mathrm{H} \rightarrow \mathrm{OH}+\mathrm{O}$ is half that of reaction $\mathrm{H}+\mathrm{O}_{2}+\mathrm{M} \rightarrow \mathrm{HO}_{2}+\mathrm{M}$. For syngas mixtures, however, the crossover variable is diminished by $\left(1-\delta_{\mathrm{O}}\right)$, which tends to increase the crossover temperature, since $\alpha$ is an increasing function of temperature. The evolution of this newly defined crossover temperature is shown in Fig. 4. Besides the well-known increase with pressure arising from the third-body concentration [17], the results in the figure reveal that the variation associated with the syngas composition is important only for fuel mixtures with very low $\mathrm{H}_{2} /\left(\mathrm{H}_{2}+\mathrm{CO}\right)$ values (below $20 \%$ in volume), which, however, may often be encountered in practical applications, such as those involving blast-furnace gas [20].

The separate branched-chain-explosion descriptions given below will account for the markedly different composition of the radical pool found for $\alpha>1$ and $\alpha<1$. This composition is determined by the normalized form $\left(V_{\mathrm{H}}, V_{\mathrm{O}}, V_{\mathrm{OH}}, V_{\mathrm{HO}_{2}}, V_{\mathrm{H}_{2} \mathrm{O}_{2}}\right) /\left(V_{\mathrm{H}}+V_{\mathrm{O}}+V_{\mathrm{OH}}+V_{\mathrm{HO}_{2}}+V_{\mathrm{H}_{2} \mathrm{O}_{2}}\right)$ of the eigenvector $\bar{V}$ associated with the dominant eigenvalue, which is used in Fig. 5 to illustrate the dependence on temperature of the radical-pool content for two different $\mathrm{H}_{2}$-CO-air stoichiometric mixtures at atmospheric pressure. It is clear that, at high temperatures, $\mathrm{H}, \mathrm{O}$, and $\mathrm{OH}$ are the main radicals responsible for ignition, while $\mathrm{HO}_{2}$ and $\mathrm{H}_{2} \mathrm{O}_{2}$ are dominant at low temperature, thereby justifying the two different analyses that follow. 


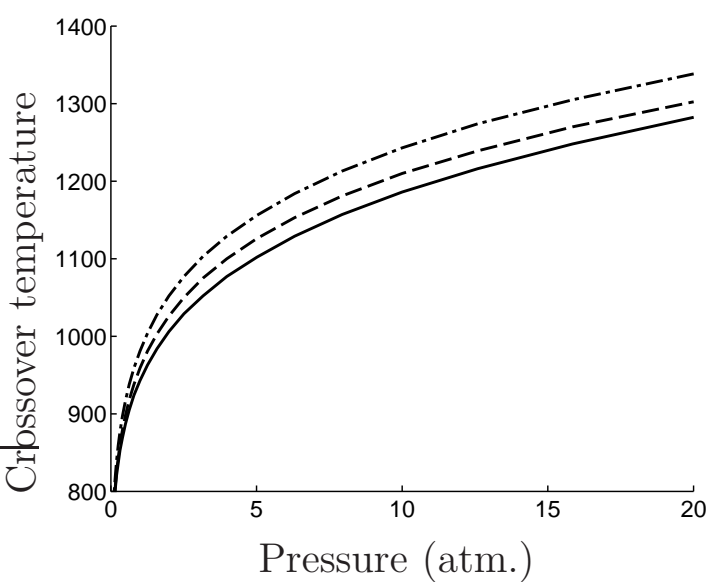

Figure 4: The variation with pressure of the crossover temperature, defined by the condition $\alpha=1$, for a stoichiometric syngas-air mixture with $\mathrm{H}_{2}: 100 / \mathrm{CO}: 0$ (solid curve), $\mathrm{H}_{2}: 10 / \mathrm{CO}: 90$ (dashed curve), and $\mathrm{H}_{2}: 5 / \mathrm{CO}: 95$ (dot-dashed curve).

\subsection{Chain-branching below crossover}

For temperatures below crossover, recombination through steps 4 and 12 keep the radicals $\mathrm{H}, \mathrm{O}$, and $\mathrm{OH}$ in steady state, with associated small concentrations that can be obtained in terms of those of $\mathrm{HO}_{2}$ and $\mathrm{H}_{2} \mathrm{O}_{2}$ by equating to zero their production rates, given by the first three rows in the linear branching equation (1), to give

$$
\begin{aligned}
& -\left(l_{1}+l_{4}\right) C_{\mathrm{H}}+l_{2} C_{\mathrm{O}}+\left(l_{3}+l_{9}\right) C_{\mathrm{OH}}+l_{7} C_{\mathrm{HO}_{2}}+\omega_{5}=0 \\
& l_{1} C_{\mathrm{H}}-\left(l_{2}+l_{12}\right) C_{\mathrm{O}}+\omega_{11}=0 \\
& l_{1} C_{\mathrm{H}}+l_{2} C_{\mathrm{O}}-\left(l_{3}+l_{9}\right) C_{\mathrm{OH}}+l_{10} C_{\mathrm{HO}_{2}}+2 l_{8} C_{\mathrm{H}_{2} \mathrm{O}_{2}}=0 .
\end{aligned}
$$

Adding (11) and (13) to eliminate $C_{\mathrm{OH}}$ and using (12) to eliminate $C_{\mathrm{O}}$ in the resulting equation leads to

$$
k_{4} C_{\mathrm{O}_{2}} C_{\mathrm{M}_{4}} C_{\mathrm{H}}=\left[\left(l_{7}+l_{10}\right) C_{\mathrm{HO}_{2}}+2 l_{8} C_{\mathrm{H}_{2} \mathrm{O}_{2}}+\omega_{5}+2\left(1-\delta_{\mathrm{O}}\right) \omega_{11}\right] /(1-\alpha)
$$

as an expression for the $\mathrm{H}$-atom recombination rate, where $\delta_{\mathrm{O}}$ and $\alpha$ are defined in (9) and (10), respectively. This last equation can be used in (11) 
Figure 5: Radical-pool composition obtained from the normalized eigenvector associated with the dominant eigenvalue for $p=1 \mathrm{~atm}, \phi=1$ with $90 \% \mathrm{H}_{2}, 10 \% \mathrm{CO}$ (upper plot) and $10 \% \mathrm{H}_{2}, 90 \% \mathrm{CO}$ (lower plot).

to give the simplified branching problem

$$
\left[\begin{array}{c}
\frac{\mathrm{d} C_{\mathrm{HO}_{2}}}{\mathrm{~d} t} \\
\frac{\mathrm{d} C_{\mathrm{H}_{2} \mathrm{O}_{2}}}{\mathrm{~d} t}
\end{array}\right]=\left[\begin{array}{cc}
\frac{\alpha\left(l_{7}+l_{10}\right)}{1-\alpha} & \frac{2 l_{8}}{1-\alpha} \\
l_{7} & -l_{8}
\end{array}\right] \cdot\left[\begin{array}{c}
C_{\mathrm{HO}_{2}} \\
C_{\mathrm{H}_{2} \mathrm{O}_{2}}
\end{array}\right]+\left[\begin{array}{c}
\frac{\epsilon-}{1-\alpha} \\
0
\end{array}\right]
$$

where

$$
\epsilon_{-}=(2-\alpha) \omega_{5}+2\left(1-\delta_{\mathrm{O}}\right) \omega_{11}
$$

is a measure of the reduced initiation rate. The associated characteristic equation for the Jacobian matrix in (15) can be solved to give

$$
\lambda_{-}=\frac{\left\{\left[(1-\alpha) l_{8}+\alpha\left(l_{7}+l_{10}\right)\right]^{2}+8(1-\alpha) l_{7} l_{8}\right\}^{1 / 2}+\alpha\left(l_{7}+l_{10}\right)-(1-\alpha) l_{8}}{2(1-\alpha)}
$$


for the largest eigenvalue, which produces the dominant contribution for sufficient large times $\lambda_{-} t \gg 1$, yielding the approximate solution

$$
C_{\mathrm{HO}_{2}}=\frac{\epsilon_{-}}{(1-\alpha) \lambda_{-}} e^{\lambda_{-} t}
$$

for the hydroperoxyl concentration.

The criterion used in (88) to define the duration of the branching stage leads in this case to the expression

$$
t_{-}=\lambda_{-}{ }^{-1} \ln \left[(1-\alpha) \lambda_{-}^{2} /\left(2 k_{6} \epsilon_{-}\right)\right],
$$

as can be seen by equating the $\mathrm{HO}_{2}$ production rate

$$
\frac{\mathrm{d} C_{\mathrm{HO}_{2}}}{\mathrm{~d} t}=\lambda_{-} C_{\mathrm{HO}_{2}},
$$

consistent with (18), to its consumption rate $2 k_{6} C_{\mathrm{HO}_{2}}^{2}$ by step 6 , with $C_{\mathrm{HO}_{2}}$ evaluated from (18). The resulting expression applies below crossover, i.e. for $\alpha<1$, but fails as crossover is approached, because $\lambda_{-}^{-1} \propto(1-\alpha)$, as seen in (17), so that the predicted branched-chain-explosion duration vanishes as $\alpha$ tends to one. The prediction derived here is plotted in Fig. 3 ,

\subsection{Chain-branching above crossover}

For temperatures above crossover, the radical pool is dominated by $\mathrm{H}$, $\mathrm{O}$, and $\mathrm{OH}$, while the concentrations of $\mathrm{HO}_{2}$ and $\mathrm{H}_{2} \mathrm{O}_{2}$ are negligibly small, resulting in a chain-branching equation (11) involving the reduced radicalconcentration vector $\bar{C}=\left(C_{\mathrm{H}}, C_{\mathrm{O}}, C_{\mathrm{OH}}\right)$ with associated Jacobian matrix

$$
\mathbf{A}=\left[\begin{array}{ccc}
-l_{1}-l_{4} & l_{2} & l_{3}+l_{9} \\
l_{1} & -l_{2}-l_{12} & 0 \\
l_{1} & l_{2} & -l_{3}-l_{9}
\end{array}\right]
$$


and associated initiation-rate vector $\bar{\epsilon}=\left(\omega_{5}, \omega_{11}, 0\right)$. As shown in [15] for hydrogen, the resulting cubic characteristic equation has a single positive real root $\lambda^{+}$, whose value can be determined with excellent accuracy by neglecting the cubic term to give

$$
\lambda_{+}=\frac{2 b_{0}}{\left(b_{1}^{2}+4 b_{0} b_{2}\right)^{1 / 2}+b_{1}},
$$

where

$$
\begin{aligned}
& b_{0}=2 l_{1} l_{2}\left(l_{3}+l_{9}\right)(\alpha-1) / \alpha \\
& b_{1}=\left(l_{2}+l_{4}+l_{12}\right)\left(l_{3}+l_{9}\right)+l_{1}\left(2 l_{2}+\alpha l_{12}\right) / \alpha \\
& b_{2}=l_{1}+l_{2}+l_{3}+l_{4}+l_{9}+l_{12}
\end{aligned}
$$

are the coefficients of the characteristic polynomial.

The explicit expression (22) can be used to evaluate the branching rate $\lambda_{+}$for given conditions of composition, pressure and temperature. Unfortunately, a simple explicit description is not available for the associated coefficient $a_{+}$appearing in the reduced radical-pool description $\left(C_{\mathrm{H}}, C_{\mathrm{O}}, C_{\mathrm{OH}}\right)=$ $a_{+} \bar{V}_{+} e^{\lambda_{+} t}$, needed to determine the branching-stage duration $t_{B}$. This difficulty can be overcome, following [15], by considering the branching equation

$$
\frac{\mathrm{d} C_{\mathrm{H}}}{\mathrm{d} t}=(\alpha-1) l_{4} C_{\mathrm{H}}+\epsilon_{+},
$$

where

$$
\epsilon_{+}=\omega_{5}+2\left(1-\delta_{\mathrm{O}}\right) \omega_{11}
$$

Equation 23 is derived from (11)-(4) by neglecting reactions 7, 8, and 10, which are proportional to $C_{\mathrm{HO}_{2}}$ and $C_{\mathrm{H}_{2} \mathrm{O}_{2}}$, and assuming $\mathrm{O}$ and $\mathrm{OH}$ to be in steady state. While (23) is only accurate for extremely rich mixtures [15], the alternative equation

$$
\frac{\mathrm{d} C_{\mathrm{H}}}{\mathrm{d} t}=\lambda_{+} C_{\mathrm{H}}+\epsilon_{+}
$$


obtained by replacing the branching rate $(\alpha-1) l_{4}$ with the value $\lambda_{+}$given in (22), leads to an accurate description for the H-atom variation with time, given for $\lambda_{+} t \gg 1$ by

$$
C_{\mathrm{H}}=\frac{\epsilon_{+}}{\lambda_{+}} e^{\lambda_{+} t}
$$

with accompanying $\mathrm{O}$ and $\mathrm{OH}$ concentrations obtained from the steady-state expressions $C_{\mathrm{O}}=\left[l_{1} /\left(l_{2}+l_{12}\right)\right] C_{\mathrm{H}}$ and $C_{\mathrm{OH}}=\left[l_{1}\left(2 l_{2}+l_{12}\right) /\left(l_{2}+l_{12}\right)\right] C_{\mathrm{H}}$. This exponential radical growth continues until the backward rates of the shuffle reactions 1-3 become significant. Correspondingly, the branching time can be computed, following [11], as the time at which the shuffle reaction 1 reaches partial equilibrium. Our computations revealed that for all conditions tested here the resulting branching times are very close to those obtained with use of (8), based instead on the achievement of $\mathrm{HO}_{2}$ steady state through its consumption by $2 \mathrm{HO}_{2} \rightarrow \mathrm{H}_{2} \mathrm{O}_{2}+\mathrm{O}_{2}$, which is the criterion to be employed below.

The $\mathrm{HO}_{2}$ production and consumption rates can be evaluated with use made of the approximate expression $C_{\mathrm{HO}_{2}}=C_{\mathrm{H}} /(\alpha-1)$, which follows from the fact that the growth rate of $C_{\mathrm{H}}$ is proportional to $(\alpha-1) l_{4} C_{\mathrm{H}}$ in (23), while that of $\mathrm{HO}_{2}$ is proportional to $l_{4} C_{\mathrm{H}}$, as can be inferred from (3). Equating the $\mathrm{HO}_{2}$ production rate, $\mathrm{d} C_{\mathrm{HO}_{2}} / \mathrm{d} t=(\alpha-1)^{-1} \mathrm{~d} C_{\mathrm{H}} / \mathrm{d} t=\lambda_{+} C_{\mathrm{H}} /(\alpha-1)$, to its consumption rate by step $6,2 k_{6} C_{\mathrm{HO}_{2}}^{2}=2 k_{6} C_{\mathrm{H}}^{2} /(1-\alpha)^{2}$, with $C_{\mathrm{H}}$ evaluated from (26) , leads to the explicit prediction

$$
t_{+}=\lambda_{+}{ }^{-1} \ln \left[(\alpha-1) \lambda_{+}^{2} /\left(2 k_{6} \epsilon_{+}\right)\right]
$$

for the duration of the branching stage when the initial temperature lies above crossover. Since $\lambda_{+}$shows a linear dependence on $\alpha-1$ through the 
coefficient $b_{0}$ in (22), the resulting predicted duration (27) diverges as the crossover temperature is approached, thereby emphasizing that the analytical result (27) is valid only above crossover. This prediction also is plotted in Fig. 通,

\subsection{A uniformly valid expression for $t_{B}$}

As shown in Fig. 6, away from crossover the approximate expressions given in (17) and (22) for $\lambda_{-}$and $\lambda_{+}$provide an accurate representation for the branching rate $\lambda$, corresponding to the largest eigenvalue of the chainbranching matrix A, but they diverge as crossover is approached, i.e. for $|\alpha-1| \ll 1$, so that neither $\lambda_{-}$nor $\lambda_{+}$provides sufficient accuracy in a small temperature range about crossover. The investigation of the transition between the low-temperature and high-temperature branching regimes requires consideration of conditions near crossover. The analysis provides a uniformly valid description for $\lambda$, enabling a prediction for the duration of the branched-chain explosion through (8) that can be used regardless of the initial temperature, as shown below.

As discussed in [21], chain-branching explosions around crossover can be investigated using $\mathrm{H}$ and $\mathrm{HO}_{2}$ as the only branching intermediates, with all other radicals assumed to be in steady state. The associated Jacobian matrix

$$
\mathbf{A}=\left[\begin{array}{cc}
l_{4}(\alpha-1) & 3 l_{7}+l_{10} \\
l_{4} & -l_{7}-l_{10}
\end{array}\right],
$$

obtained from (5) by introduction of steady states for $\mathrm{O}, \mathrm{OH}$, and $\mathrm{H}_{2} \mathrm{O}_{2}$, has two eigenvalues, the larger of which

$$
\lambda_{t}=\left[\left(c_{1}^{2}+4 c_{0}\right)^{1 / 2}-c_{1}\right] / 2
$$




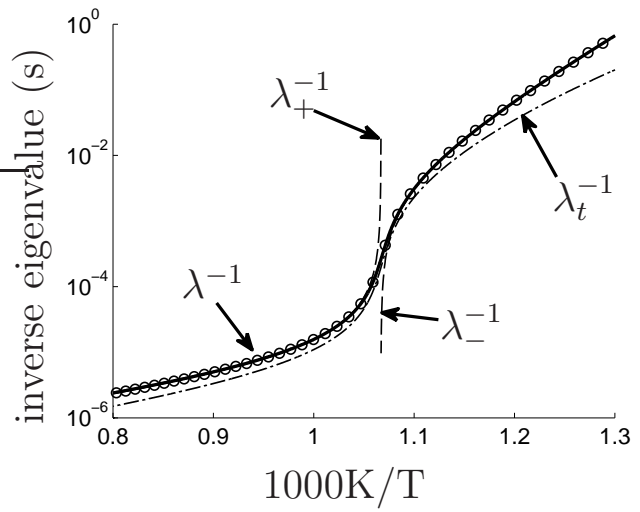

Figure 6: The variation of the branching rate for a stoichiometric syngas mixture with $\mathrm{H}_{2}: \mathrm{CO}: 50: 50$ at $p=1 \mathrm{~atm}$ as obtained numerically from the characteristic equation associated with (3) (thick solid curve), from evaluations of the expressions given in (17) and (22) (dashed curves), (29) (dot-dashed curve), and (33) (circles).

determines the branching rate in this transitional regime, with $c_{0}=l_{4}[(2+$ $\left.\alpha) l_{7}+\alpha l_{10}\right]$ and $c_{1}=l_{7}+l_{10}-l_{4}(\alpha-1)$ representing the coefficients of the characteristic polynomial. As can be seen in the comparison of Fig. 6, the expression (29) provides an accurate representation for the branching rate around crossover, but displays increasing errors both below and above crossover, where $\lambda_{t}$ fails to approach the correct asymptotic values $\lambda_{-}$and $\lambda_{+}$. The reason for these inaccuracies lies in the approximations involved in (28). Thus, sufficiently above crossover, where $\mathrm{HO}_{2}$ tends to become unimportant, Eq. (28) leads to a branching rate $\lambda_{t}=l_{4}(\alpha-1)$ in agreement with the approximation (23), different from the value $\lambda=\lambda_{+}$applying at these high-temperature conditions. On the other hand, below crossover, where $\mathrm{H}$ approaches steady state with a concentration given by $C_{\mathrm{H}}=\left(3 l_{7}+l_{10}\right) C_{\mathrm{HO}_{2}} /\left[(1-\alpha) l_{4}\right]$, the matrix (28) simplifies to give the reduced branching rate $\lambda_{t}=\left[l_{7}(2+\alpha)+\alpha l_{10}\right] /(1-\alpha)$, different from the value $\lambda_{-}$appearing in (20). 
These deficiencies can be addressed by introducing the non-zero, nondiverging branching ratios

$$
\begin{aligned}
& \Lambda_{+}=\lambda_{+} /\left[l_{4}(\alpha-1)\right] \\
& \Lambda_{-}=\lambda_{-} \frac{1-\alpha}{l_{7}(2+\alpha)+\alpha l_{10}}
\end{aligned}
$$

in the definition of a modified branching matrix

$$
\mathbf{A}=\left[\begin{array}{cc}
(\alpha-1) l_{4} \Lambda_{+} & \left(3 l_{7}+l_{10}\right) \Lambda_{+} \\
l_{4} \Lambda_{-} & \left(-l_{7}-l_{10}\right) \Lambda_{-}
\end{array}\right],
$$

which displays the appropriate behavior away from crossover, correcting both the $\mathrm{H}$-dominated high-temperature path through $\Lambda_{+}$and the $\mathrm{HO}_{2}$-dominated low-temperature path through $\Lambda_{-}$, while maintaining the general form (28). The resulting branching rate

$$
\lambda=\left[\left(C_{1}^{2}+4 C_{0}\right)^{1 / 2}-C_{1}\right] / 2
$$

involving now the coefficients

$$
\begin{aligned}
& C_{0}=l_{4}\left[(2+\alpha) l_{7}+\alpha l_{10}\right] \Lambda_{+} \Lambda_{-} \\
& C_{1}=\left(l_{7}+l_{10}\right) \Lambda_{-}-l_{4}(\alpha-1) \Lambda_{+},
\end{aligned}
$$

provides sufficient accuracy, as can be seen in the sample comparison of Fig. 6. Additional tests were run for different initial compositions, temperatures, and pressures to show that the prediction (33) consistently differs by less than $2 \%$ from the value of $\lambda$ obtained by numerical solution of the characteristic equation associated with (5).

The branching rate (33) can be used in writing the expression

$$
t_{B}=\lambda^{-1} \ln \left[(1+\alpha) \lambda^{2} /\left(2 k_{6} \epsilon\right)\right],
$$


for the duration of the chain-branching stage, consistent with the functional form of the low-temperature and high-temperature predictions given in (19) and (27). The factor $(1+\alpha)$ and the initiation parameter

$$
\epsilon=\epsilon_{+}=\omega_{5}+2\left(1-\delta_{\mathrm{O}}\right) \omega_{11}
$$

have been selected to ensure that the expression (34) reduces to (19) for $\alpha \ll 1$ and to (27) for $\alpha \gg 1$, thereby providing an accurate prediction for all temperatures. This is illustrated in Fig. 3, where the predictions obtained from (34) are seen to be indistinguishable from those obtained from the complete expression (8).

\section{The thermal explosion below crossover}

As seen in Fig. 2, ignition below crossover proceeds as a two-stage process, with a thermal explosion following the initial branched-chain period investigated above. This thermal explosion has been analyzed for hydrogen-air

mixtures in [12], with the activation energy of the rate-controlling reactions used as an asymptotically large parameter in deriving an explicit expression for the explosion time. The results were employed to derive expressions for the third explosion limit in closed containers [22], giving results in close agreement with those of detailed chemistry. The analysis given below extends our previous work by accounting for the modifications to the chemistry arising in the presence of $\mathrm{CO}$.

During the thermal explosion, developing with small temperature increments and associated negligible reactant consumption, the radicals $\mathrm{H}, \mathrm{O}, \mathrm{OH}$, and $\mathrm{HO}_{2}$ maintain steady states, enabling a three-step reduced mechanism 
to be derived from the skeletal mechanism shown in Table 1, with overall reactions

$$
\begin{gathered}
\mathrm{H}_{2}+\mathrm{O}_{2} \stackrel{\text { I }}{\rightarrow} \mathrm{H}_{2} \mathrm{O}_{2} \\
2 \mathrm{H}_{2}+\mathrm{O}_{2} \stackrel{\text { II }}{\rightarrow} 2 \mathrm{H}_{2} \mathrm{O} \\
2 \mathrm{CO}+\mathrm{O}_{2} \stackrel{\text { III }}{\rightarrow} 2 \mathrm{CO}_{2},
\end{gathered}
$$

and associated rates that can be written in the simplified form

$$
\begin{aligned}
\omega_{\mathrm{I}} & =\frac{2-\alpha}{2(1-\alpha)} \omega_{7}+\frac{\alpha}{2(1-\alpha)} \omega_{10} \\
\omega_{\mathrm{II}} & =\frac{\left(1-\delta_{\mathrm{OH}}\right)}{2(1-\alpha)}\left(\omega_{10}+2 \omega_{8}\right) \\
\omega_{\mathrm{III}} & =\frac{\left(1+\delta_{\mathrm{OH}}\right)}{2(1-\alpha)} \omega_{10}+\frac{\delta_{\mathrm{OH}}}{(1-\alpha)} \omega_{8}
\end{aligned}
$$

involving the parameter

$$
\delta_{\mathrm{OH}}=\frac{k_{9} C_{\mathrm{CO}}}{k_{3} C_{\mathrm{H}_{2}}+k_{9} C_{\mathrm{CO}}}
$$

representing the fraction of radicals $\mathrm{OH}$ consumed by carbon monoxide, a quantity that is assumed to be constant during the ignition event. The above expressions involve the elementary-reaction rates

$$
\omega_{7}=k_{7} C_{\mathrm{H}_{2}} C_{\mathrm{HO}_{2}}, \quad \omega_{8}=k_{8} C_{\mathrm{M}_{8}} C_{\mathrm{H}_{2} \mathrm{O}_{2}}, \quad \text { and } \quad \omega_{10}=k_{10} C_{\mathrm{CO}} C_{\mathrm{HO}_{2}}
$$

where $C_{\mathrm{HO}_{2}}$ is evaluated, following [12], with the simplified steady-state expression

$$
C_{\mathrm{HO}_{2}}=\sqrt{\frac{k_{8} C_{\mathrm{M}_{8}} C_{\mathrm{H}_{2} \mathrm{O}_{2}}}{k_{6}(1-\alpha)}},
$$

derived by neglecting contributions from the elementary reactions 7 and 10, an excellent approximation under most conditions [12].

With reactant consumption neglected, the homogeneous ignition history associated with the above reduced chemistry can be obtained by integration of

$$
\begin{aligned}
\frac{\mathrm{d} C_{\mathrm{H}_{2} \mathrm{O}_{2}}}{\mathrm{~d} t} & =\omega_{\mathrm{I}} \\
\rho c_{p} \frac{\mathrm{d} T}{\mathrm{~d} t} & =-2 h_{\mathrm{H}_{2} \mathrm{O}} \omega_{\mathrm{II}}-2\left(h_{\mathrm{CO}_{2}}-h_{\mathrm{CO}}\right) \omega_{\mathrm{III}}
\end{aligned}
$$


with initial temperature $T=T_{o}$ and initial $\mathrm{H}_{2} \mathrm{O}_{2}$ concentration $C_{\mathrm{H}_{2} \mathrm{O}_{2}}=0$, the concentration of $\mathrm{H}_{2} \mathrm{O}_{2}$ produced in the earlier branching stage being negligible here. In the formulation $\rho$ and $c_{p}$ are the initial values of the density and specific heat at constant pressure. The heat released by reaction I has been neglected in the energy equation, since its contribution is small compared with that of the other two reactions, which have been expressed in terms of the enthalpies of formation $h_{\mathrm{H}_{2} \mathrm{O}}=-241.8 \mathrm{~kJ} / \mathrm{mol}, h_{\mathrm{CO}_{2}}=-393.5 \mathrm{~kJ} / \mathrm{mol}$, and $h_{\mathrm{CO}}=-110.5 \mathrm{~kJ} / \mathrm{mol}$.

The analysis proceeds by noting that the activation energies of the overall reaction-rate constants $k_{8}, k_{7}\left(k_{8} / k_{6}\right)^{1 / 2}$, and $k_{10}\left(k_{8} / k_{6}\right)^{1 / 2}$ appearing in (40) upon substitution of (41) are very similar, so that a single dimensionless activation energy $\beta=E_{8} /\left(R^{o} T_{o}\right)+n_{8}$, based for definiteness on the low-pressure rate parameters $n_{8}=-4.20$ and $E_{8}=213.71 \mathrm{~kJ} / \mathrm{mol}$ of the elementary rate constant $k_{8}=A_{8} T^{n 8} \exp \left[-E_{8} /\left(R^{o} T\right)\right]$, characterizes the strong temperature dependence of all three rates $\omega_{7}, \omega_{8}$, and $\omega_{10}$. For large values of $\beta$, introduction of the rescaled variables

$$
\theta=\beta \frac{T-T_{o}}{T_{o}}, \quad \varphi=(\beta q X)^{2 / 3} \frac{C_{\mathrm{H}_{2} \mathrm{O}_{2}}}{C_{\mathrm{M}_{8}}}, \quad \text { and } \quad \tau=\frac{(\beta q)^{1 / 3}}{(1-\alpha)} k_{8} C_{\mathrm{M}_{8}} X^{-2 / 3} t,
$$

reduces the problem to the integration of

$$
\begin{aligned}
& \frac{\mathrm{d} \varphi}{\mathrm{d} \tau}=\varphi^{1 / 2} e^{\theta} \\
& \frac{\mathrm{d} \theta}{\mathrm{d} \tau}=\varphi e^{\theta}+\Omega \varphi^{1 / 2} e^{\theta}
\end{aligned}
$$

with initial conditions $\varphi(0)=\theta(0)=0$. The constant $k_{8}$ in the definition of $\tau$ and the reaction-rate parameter

$$
X=\frac{2 C_{\mathrm{M}_{8}} \sqrt{k_{8} k_{6}(1-\alpha)}}{(2-\alpha) k_{7} C_{\mathrm{H}_{2}}+\alpha k_{10} C_{\mathrm{CO}}}
$$

are to be evaluated at the initial temperature. The above expressions involve the dimensionless effective heat of reaction

$$
q=\left(1-\delta_{\mathrm{OH}}\right) q_{\mathrm{II}}+\delta_{\mathrm{OH}} q_{\mathrm{III}}
$$

based on the heat-release parameters

$$
q_{\mathrm{II}}=\frac{-2 h_{\mathrm{H}_{2} \mathrm{O}} C_{\mathrm{M}_{8}}}{\rho c_{p} T_{o}} \quad \text { and } \quad q_{\mathrm{III}}=\frac{-2\left(h_{\mathrm{CO}_{2}}-h_{\mathrm{CO}}\right) C_{\mathrm{M}_{8}}}{\rho c_{p} T_{o}} \text {, }
$$

with $q$ defined such that $q$ tends to $q_{\mathrm{II}}$ for low-CO syngas content and to $q_{\mathrm{III}}$ for high-CO syngas content. 
The thermal explosion depends on the single parameter

$$
\Omega=\left(1+q_{\mathrm{III}} / q\right) \frac{(\beta q)^{1 / 3} X^{-2 / 3} k_{10} C_{\mathrm{CO}}}{\left[(2-\alpha) k_{7} C_{\mathrm{H}_{2}}+\alpha k_{10} C_{\mathrm{CO}}\right]},
$$

appearing in (45). Dividing (45) by (44) provides $\theta=\frac{2}{3} \varphi^{3 / 2}+\Omega \varphi$ upon integration, leading to the dimensionless thermal-explosion time

$$
\tau_{E}=\int_{0}^{\infty} \frac{\mathrm{d} \varphi}{\varphi^{1 / 2} \exp \left(\frac{2}{3} \varphi^{3 / 2}+\Omega \varphi\right)}
$$

after substitution into (44) and subsequent integration. This integral takes the limiting values

$$
\tau_{E}=(2 / 3)^{2 / 3} \Gamma(1 / 3) \simeq 2.0444
$$

for $\Omega \ll 1$ and

$$
\tau_{E}=\int_{0}^{\infty} \frac{\mathrm{d} \varphi}{\varphi^{1 / 2} \exp (\Omega \varphi)}=\sqrt{\pi / \Omega}
$$

for $\Omega \gg 1$. The combined expression

$$
\tau_{E}=\left(\Omega / \pi+2.044^{-2}\right)^{-1 / 2}
$$

provides sufficient accuracy for all values of $\Omega$, as can be seen from the comparison shown in Fig. 17. The definition of the dimensionless time $\tau$ given in (43) can be used to show that the dimensional explosion time is

$$
t_{E}=\frac{X^{2 / 3}(1-\alpha)}{(\beta q)^{1 / 3} k_{8} C_{\mathrm{M}_{8}}} \tau_{E} .
$$

\section{Evaluations of the analytical predictions}

An expression for the branching time has been derived in Section 3, and an expression for the thermal-explosion time has been derived in Section 4. Since the branching stage is essentially completed before the beginning of the explosion stage, an accurate estimate of the ignition delay may be expected to be provided by the sum of these two times. A representative test of that accuracy is shown in Fig. 8 for $p=10 \mathrm{~atm}$, a value between limiting pressures of practical interest for different problems. As may be inferred from this figure, the value of $t_{E}$ vanishes at crossover, found to occur at $T \simeq 1200 \mathrm{~K}$ at this elevated pressure, a result that can be anticipated readily from the presence of the factor $(1-\alpha)$ in (54), and it increases rapidly with decreasing temperature, that being a consequence of the large activation energy of the controlling rates $\omega_{7}$ and $\omega_{8}$. The comparison of $t_{B}$ 
Figure 7: The value of $\tau_{E}$ determined from (50) (solid curve) and by evaluation of the approximate expression (53) (dashed curve); the dot-dashed curve shows the relative error.

and $t_{E}$, the former evaluated from (34) with use made of (30) and (33)-(35), reveals that the duration of the thermal explosion near crossover is small compared with that of the chain-branching stage. Both times are equal at $T \simeq 1075 \mathrm{~K}$, and for lower temperatures the thermal explosion dominates the ignition history, with the approximation $t_{i}=t_{E}$ becoming increasingly accurate away from crossover. The figure also indicates that the sum $t_{B}+t_{E}$ of the durations of the chain-branching stage and of the thermal-explosion stage provides a very accurate prediction for the induction time at all temperatures.

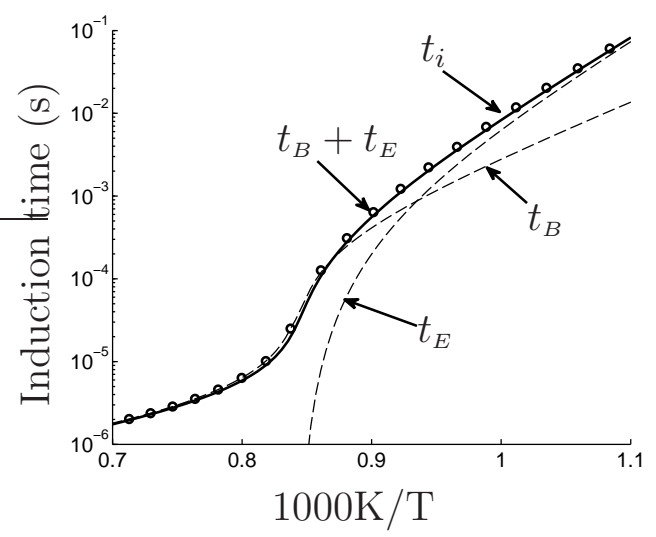

Figure 8: The solid curve represents the variation with temperature of the induction time $t_{i}$ obtained numerically with detailed chemistry for a stoichiometric syngas-air mixture with $90 \% \mathrm{H}_{2}$ and $10 \% \mathrm{CO}$ at $p=10$ atm. The dashed curves are evaluated from (34) and (54) for those same conditions, with the circles representing the sum $t_{B}+t_{E}$.

The prediction $t_{i}=t_{B}+t_{E}$ is further tested in Fig. 9, where $t_{B}$ is evaluated 
from (34) with use made of (30) and (33)-(35), and $t_{E}$ is evaluated from (54) with the approximate formula (53) for $\tau_{E}$. These comparisons, which span the full range of conditions of interest, reveal that the differences usually are nearly imperceptible. The top pair of figures, which cover the complete range of possible hydrogen content, show the expected increase in ignition delay with decreasing hydrogen mole fractions at low values, as the crossover temperature increases, as well as a possibly unexpected decrease with increasing hydrogen as its mole fraction approaches unity for the lower temperatures. This last effect is a consequence of the larger heat release in $\mathrm{CO}$ oxidation shortening the explosion time as the $\mathrm{CO}$ concentration increases. The ignition delay is seen in the middle pair of figures to depend only very weakly on the equivalence ratio over a wide range of values, while from the bottom pair, where the solid curves correspond to those in Fig. 1, it is seen to exhibit an interesting variation with pressure associated with the increase of the crossover temperature with increasing pressure. For example, the delay at 1 atm is greater than that at higher pressures for both high and low temperatures, but at intermediate temperatures it is less because the system has moved above crossover there. The analytical results aid in understanding this nonmonotonic type of behavior.

\section{Conclusions}

This investigation has been focused solely on chemical-kinetic influences on ignition delays. Although that is only one of many combustion properties of interest, it is a very important one for syngas, especially in gas-turbine applications, where excess hydrogen content may promote unacceptable flashback and pre-ignition. While there are syngas mixtures having high contents of hydrocarbons that would necessitate considerations of extensive detailed chemical-kinetic mechanisms, the hydrocarbon contents of many such mixtures are in single-digit percentages, enabling attention to be restricted to mixtures of $\mathrm{H}_{2}$ and $\mathrm{CO}$ with inerts for investigating most of their combustion processes. Even then, however, on the order of 30 reversible elementary chemical-kinetic steps need to be considered, which sometimes makes computations prohibitively complicated. The first important finding of the present study is that for addressing ignition delays only 12 irreversible elementary steps have to be considered. This is a significant reduction that, in itself, may facilitate computations that otherwise could not be accomplished.

Since situations arise in which computations with elementary chemistry are beyond the realm of practical consideration, the availability of analytic formulas for ignition delays can help calculations to be made simply, without extensive computation. Such formulas also help to reveal the controlling parameters and chemical processes involved, thereby increasing understanding. The present investigation 

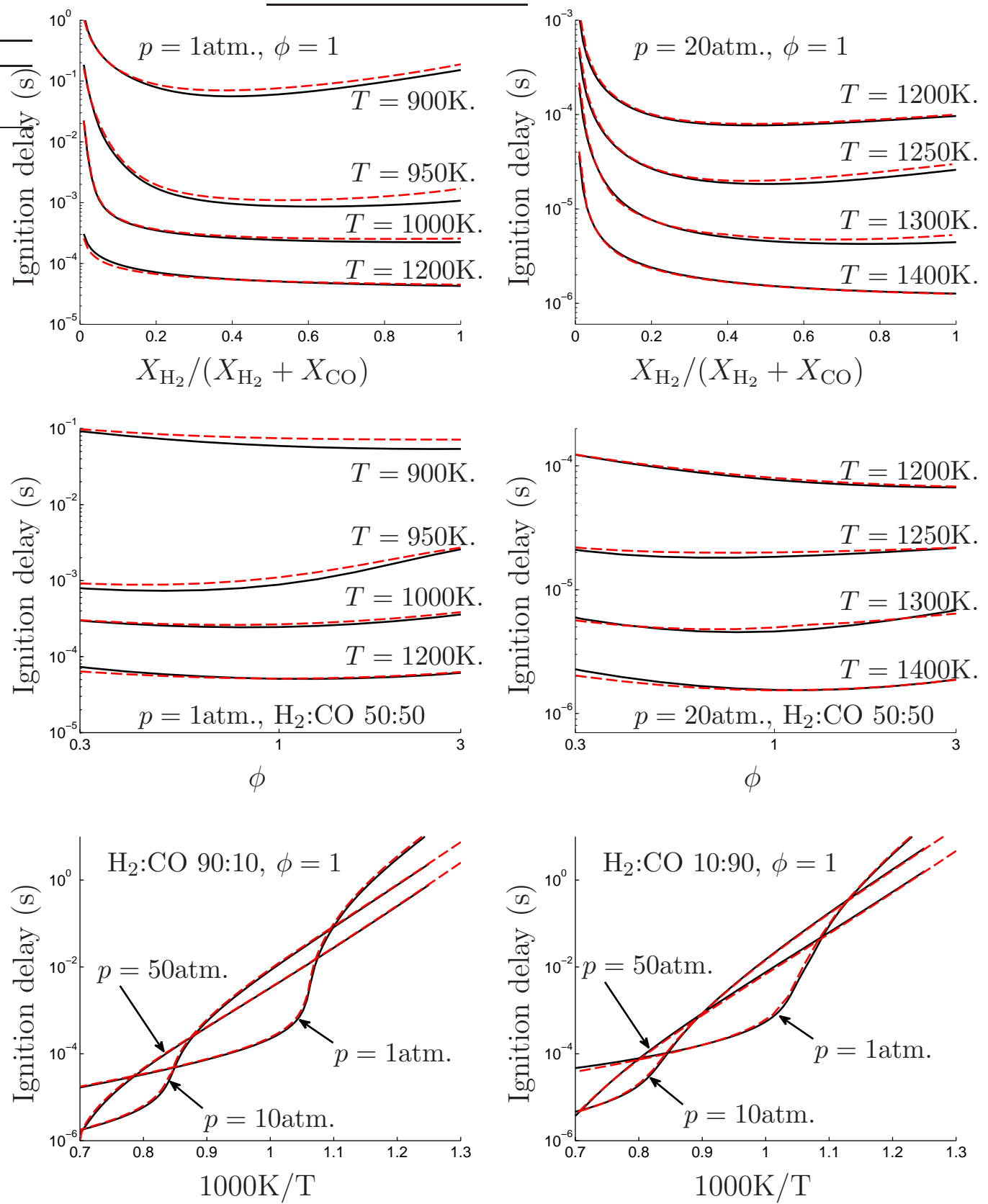

Figure 9: Comparison of ignition delays $t_{i}$ in air obtained by numerical integrations for the complete 30-step chemistry (solid curves) with the analytical prediction $t_{i}=t_{B}+t_{E}$ (dashed curves), having $t_{E}=0$ for $\alpha \geq 1$. 
has developed these formulas for ignition delays, providing accuracies essentially equivalent to those found through computations with detailed chemistry.

Building on previous work that addressed only $\mathrm{H}_{2}$, ignition-delay formulas have been extended here to include the effects of $\mathrm{CO}$ as well. It has been shown that there exists a crossover temperature that increases with increasing $\mathrm{CO}$ content, such that at temperatures above that crossover temperature autoignition occurs through a branched-chain explosion, the temperature increase being negligible during the main branching period, resulting in an accurate formula for the ignition delay at high temperatures, while in the vicinity of crossover and below, a thermal explosion process comes into play. The autoignition process in fact always involves a chain-branching process, but as the temperature decreases the duration of this process occupies a decreasing fraction of the ignition delay period with an increasing fraction of the time controlled by a thermal explosion involving autocatalytic $\mathrm{H}_{2} \mathrm{O}_{2}$ growth. Through analysis of this last process, a formula for the ignition delay has been developed here that becomes increasingly accurate as the temperature decreases. Finally, it also has been shown that the sum of the branching time and the explosion time, incorporating appropriate corrections to assure accuracy in the limits of high and low temperature, provides an excellent approximation to the total ignition delay. Autoignition times for all of these syngas mixtures may thus be calculated well from the results presented here.

\section{Acknowledgements}

This work was supported by the Spanish MCINN through project \# CSD201000010. FAW is supported by the US National Science Foundation through award \#CBET-1404026. The paper was completed at UCSD during a summer stay of PB supported by the French National Research Agency through projects Labex MEC (ANR-10-LABX-0092) and A*MIDEX (ANR-11-IDEX-0001-02).

\section{References}

\section{References}

[1] Y.S. Kim, J.J. Lee, T.S. Kim, J.L. Sohn, Effects of syngas type on the operation and performance of a gas turbine in integrated gasification combined cycle, Energ. Convers. Manage. 52 (2011) 2262-2271.

[2] M.L. Fox, G.K. Lilic, A.L. Boehman, O Le Corre, Combustion of syngas in internal combustion engines, in: T. Lieuwen, V. Yang, R. Yetter (Eds.), Synthesis Gas Combustion: Fundamentals and Applications, CRC Press, Boca Raton, FL, 2009, pp. 289-328. 
[3] G. Maschio, A. Lucchesi, G. Stoppato, Production of syngas from biomass. Bioresource Technol. 48 (1994) 119-126.

[4] S. G. Davis, A. V. Joshi, H. Wang, F. Egolfopoulos, An optimized kinetic model of $\mathrm{H}_{2} / \mathrm{CO}$ combustion, Proc. Combust. Inst. 30 (2005) 1283-1292.

[5] P. Saxena, F.A. Williams, Testing a small detailed chemicalkinetic mechanism for the combustion of hydrogen and carbon monoxide, Combust. Flame 145 (2006) 316-323. Also available at http://maeweb.ucsd.edu/ combustion/cermech/.

[6] H. Sun, S. I. Yang, G. Jomaas, C. K. Law, High-pressure laminar flame speeds and kinetic modeling of carbon monoxide/hydrogen combustion, Proc. Combust. Inst. 31 (2007) 439-446.

[7] A. Frassoldati, T. Faravelli, E. Ranzi, The ignition, combustion and flame structure of carbon monoxide/hydrogen mixtures. Note 1: Detailed kinetic modeling of syngas combustion also in presence of nitrogen compounds. Int. J. Hydrogen Energ. 32 (2007) 3471-3485.

[8] A. M. Starik, N. S. Titova, A. S. Sharipov, V. E. Kozlov, Syngas oxidation mechanism, Combust. Explo. Shock+ 46 (2010) 491-506.

[9] P. Chiesa, G. Lozza, L. Mazzocchi, Using hydrogen as a gas turbine fuel, J. Eng. Gas Turb. Power 127 (2005) 73-80.

[10] R.S. Brokaw, Ignition kinetics of the carbon monoxide-oxygen reaction, Proc. Combust. Inst. 11 (1967) 1063-1073.

[11] C. Treviño, F. Solorio, Asymptotic analysis of the high-temperature ignition of CO/H2/O2 mixtures, Combust. Flame 86 (1991) 285-295.

[12] P. Boivin, A.L. Sánchez, F.A. Williams, Explicit analytic prediction for hydrogen-oxygen ignition times at temperatures below crossover, Combust. Flame 159 (2012) 748-752.

[13] Chemical-kinetic mechanisms for combustion applications, version 2016-0815, San Diego Mechanism web page, Mechanical and Aerospace Engineering (Combustion Research), University of California at San Diego. Available at http://web.eng.ucsd.edu/mae/groups/combustion/mechanism.html.

[14] A.L. Sánchez, F. A. Williams, Corrigendum to "Recent advances in understanding of flammability characteristics of hydrogen", Prog. Ener. Combust. Sci. 54 (2016) 93-94. 
[15] P. Boivin, C. Jiménez, A.L. Sánchez, F.A. Williams, An explicit reduced mechanism for $\mathrm{H}_{2}$-air combustion, Proc. Combust. Inst., 33 (2011) 517-523.

[16] P. Boivin, A.L. Sánchez, F.A. Williams, Four-step and three-step systematically reduced chemistry for wide-range $\mathrm{H}_{2}$-air combustion problems, Combust. Flame 160 (2013) 76-82.

[17] G. von Elbe, B. Lewis, The reaction between hydrogen and oxygen above the upper explosion limit, J. Am. Chem. Soc. 59 (1937) 656-662.

[18] G. Del Alamo, F. A. Williams, A.L. Sánchez, Hydrogen-oxygen induction times above crossover temperatures, Combust. Sci. Technol. 176 (2004) 15991626 .

[19] A.L. Sánchez, F. A. Williams, Recent advances in understanding of flammability characteristics of hydrogen, Prog. Ener. Combust. Sci. 41 (2014) 1-55.

[20] M. Halmann, A. Steinfeld, Reforming of blast furnace gas with methane, steam, and lime for syngas production and $\mathrm{CO}_{2}$ capture: a thermodynamic study, Miner. Process Extr. M. 36 (2015) 7-12.

[21] X. Wang, C. K. Law, An analysis of the explosion limits of hydrogen-oxygen mixtures, J. Chem. Phys. 138 (2013) 134305.

[22] A.L. Sánchez, E. Fernández-Tarrazo, F. A. Williams, The chemistry involved in the third explosion limit of $\mathrm{H}_{2}-\mathrm{O}_{2}$ mixtures. Combust. Flame 161 (2014) $111-117$. 